\title{
Early changes in tear film protein profiles after femtosecond LASIK surgery
}

\author{
Janika Nättinen ${ }^{1 *+}\left(0\right.$, Petri Mäkinen ${ }^{1,2+}$, Ulla Aapola ${ }^{1}$, Lasse Orsila ${ }^{1}$, Juhani Pietilä ${ }^{1,2}$ and Hannu Uusitalo ${ }^{1,3}$
}

\begin{abstract}
Background: Femtosecond laser-assisted in situ keratomileusis (LASIK) has proven to be an efficacious, predictable, and safe procedure for the correction of refractive errors. We examined the early tear protein changes of patients undergoing LASIK surgery in order to better understand the mechanisms and proteins related to laser corneal surgery and initial recovery.

Methods: Corneal flaps were created with Ziemer FEMTO LDV Z6 I femtosecond laser and stroma was ablated using Wavelight EX500 excimer laser. Tear samples were collected preoperatively as well as $1.5 \mathrm{~h}$ and 1 month after LASIK treatment using glass microcapillary tubes. Relative quantification of tear proteins was performed with sequential window acquisition of all theoretical fragment ion spectra mass spectrometry (SWATH-MS).
\end{abstract}

Results: SWATH-MS revealed that 158 proteins had altered expression levels $1.5 \mathrm{~h}$ after the operation. Two-thirds of these proteins, mostly connected to migration and inflammation response, returned to preoperative levels within the first postoperative month. The other proteins, which did not return to baseline levels, included proteins connected to for example epithelial barrier function. We also identified several proteins, which correlated with surgical variables, such as the amount of correction, flap thickness and flap diameter.

Conclusions: The present study showed that an uneventful femtosecond LASIK refractive surgery induced a significant immune cell migration and inflammation-associated changes in tear proteomics profile quickly after the operation, but the expression of most proteins recovered almost completely to the preoperative levels within the first month. The individual proteins identified in our study are potential targets for the follow-up and modification of LASIK-induced biochemical processes.

Keywords: Refractive surgery, Femtosecond laser, LASIK, Tear proteomics, Wound healing

\section{Background}

Laser-assisted in situ keratomileusis (LASIK) is a safe and effective procedure for the correction of refractive errors. Therefore, it is one of the most frequently used laser eye surgery procedures and the patient satisfaction is generally high [1]. The operation is performed by creating a thin flap in the epithelium of cornea, for example with femtosecond laser, and then reshaping the

\footnotetext{
*Correspondence: janika.nattinen@tuni.fi

†Janika Nättinen and Petri Mäkinen equally contributed to this work

1 SILK, Department of Ophthalmology, Faculty of Medicine and Health Technology, Tampere University, PL 100, 33014 Tampere, Finland Full list of author information is available at the end of the article
}

underlying corneal layer (stroma). Despite the very good clinical results, there are variations in LASIK's effects and complications, which indicate individual differences in corneal and ocular surface response to surgical trauma and wound healing. Increased dry eye symptoms, reduced tear film stability and reduced corneal sensation are common after LASIK surgery, possibly due to the corneal nerve disruption and related changes on the ocular surface caused by the surgery [2-4]. However, these post-LASIK side effects are usually temporary and disappear during the first months, although a small subset of patients can develop a long term, chronic condition [5]. The depth of laser treatment, higher refractive 
correction, flap thickness, hinge parameters and sex have been identified to influence the likelihood of these side effects $[2,6,7]$.

It is known that corneal refractive surgical procedures initiate a complex cascade of healing responses in cornea and ocular surface. Corneal trauma triggers an immediate release of various cytokines and growth factors, which induce interactions between epithelial cells, keratocytes, corneal nerves, lacrimal glands, tear film, and cells of the immune system [8-11]. The tear fluid, which reflects the state of the underlying eye tissue, is not only an important part and mediator of the wound healing process, but it is also a non-invasive sampling material, making it an ideal analysis target to study the effects of ocular surgery. Therefore, we decided to analyse the early tear protein changes of patients undergoing LASIK surgery in order to better understand the mechanisms and proteins related to laser corneal surgery and initial recovery. Previously, tear proteomics evaluating the changes caused by LASIK surgery have been studied using multiplex immunoassays [12,13], isobaric tags for relative and absolute quantitation (iTRAQ) [14] and enzyme-linked immunosorbent assay (ELISA) [15, 16]. Our aim was to study the immediate protein changes caused by surgery and to identify proteins related to normal, early recovery processes after surgery by implementing the sequential window acquisition of all theoretical mass spectra (SWATH-MS) method, which enables the identification and quantification of hundreds of proteins from each individual tear sample.

\section{Methods}

\section{Study design and patients}

Seventy patients were evaluated preoperatively as well as postoperatively at $1.5 \mathrm{~h}$ and, on average, at 1 month after the surgery. Altogether 70 patients were included in the study and both eyes were initially sampled for tears. Exclusion criteria included previous eye surgery, glaucoma, dry eye symptoms and pregnancy. In proteomics analyses comparing previous visits against 1 month follow-up visit, only patients with a follow-up visit within $30 \pm 15$ days from the operation were included.

\section{Surgery protocol and clinical tests Preoperative examinations}

Before the femtosecond LASIK surgery, all patients underwent a complete preoperative ophthalmologic examination, which was performed in order to identify any severe pathology that might be a contraindication for surgery. The preoperative examination included biomicroscopy, determination of refraction, wavefront analysis (Allegro Analyzer, Wavelight AG, Erlangen, Germany) and measurements of three-dimensional corneal topography (Allegro Oculyzer, Wavelight AG, Erlangen, Germany), uncorrected and corrected distance visual acuity (UDVA and CDVA, respectively), and intraocular pressure (Nidek Tonoref RKT-7700, Gamagori, Aichi, Japan).

\section{Surgical procedure}

Prior to the surgery, various drops were instilled into the eyes. These included antibiotic levofloxacin $5 \mathrm{mg} /$ $\mathrm{ml}$ eye drops (Oftaquix, Santen Oy, Tampere, Finland), diclofenac $1 \mathrm{mg} / \mathrm{ml}$ (Voltaren Ophtha, THEA, ClermontFerrand, France) for pain and inflammation, brimonidine tartrate $2 \mathrm{mg} / \mathrm{ml}$ (Alphagan, Allergan, Westport, Ireland) for conjunctival vessel construction and oxybuprocaine hydrochloride $4 \mathrm{mg} / \mathrm{ml}$ (Oftan Obucain, Santen Oy) as a topical anesthetic. Topical medication is an essential element in refractive surgery and therefore, tear fluid changes cannot be studied without medication. An aspirating speculum (Geuder, no 15961, Heidelberg, Germany) was used to keep the eyelid open. Preoperative corneal thickness was measured with an ultrasonic pachymetry (SP-3000, Tomey Corp., Nagoya, Japan).

Femtosecond laser FEMTO LDV Z6 I (Ziemer Ophthalmic Systems, Port, Switzerland), which delivered 100 $\mathrm{nJ}$ pulse energy and $>2 \mathrm{MHz}$ repetition rate, was used for the flap creation. The target flap thickness ranged from 90 to $110 \mu \mathrm{m}$ and all flaps were roundly shaped and set at a $60-90^{\circ}$ angled edge. Plastic single-use suction rings with a $9.5 \mathrm{~mm}$ diameter were used with a target flap diameter of $9.3 \mathrm{~mm}$. The target hinge length was $4.0 \mathrm{~mm}$ in all cases. Vacuum pressure was 700 mbar and cutting time $28 \mathrm{~s}$.

The excimer laser treatment was done on the exposed stroma using the Wavelight EX500 excimer laser (Wavelight AG, Erlangen, Germany). The optical zone ranged from 6.5 to $7.0 \mathrm{~mm}$ and the treatment zone was less than $9.0 \mathrm{~mm}$. All complications during the procedure and follow-up time were recorded.

\section{Postoperative treatment}

Thirty minutes after the LASIK operation, moisture drops sodium hyaluronate $0.15 \mathrm{mg} / \mathrm{ml}$ (Oxyal, Dr Gerhard Mann Chem. -pharm. Fabrik GmbH, Berlin, Germany) were instilled into the eyes. Topical anaesthetic oxybuprocaine hydrochloride (Oftan Obucain) was instilled into the eyes one hour after the surgery. Dexamethasone and chloramphenicol containing drops (Oftan Dexa-Chlora, Santen Oy) with the tapered dose were used for the first week, starting $3 \mathrm{~h}$ after the surgery.

\section{Tear fluid collection}

Patients were asked to participate in a tear fluid collection before LASIK treatment (Vpre) and postoperatively 
$1.5 \mathrm{~h}$ after LASIK (Vpost), and at follow-up approximately 1 month after LASIK (V1m) (Fig. 1). The Vpre tear sample was taken before the installation of any eye drops. Tear samples were collected into 2 or $3 \mu \mathrm{l}$ glass microcapillary tubes and stored first at $-20^{\circ} \mathrm{C}$ and then transferred to $-80^{\circ} \mathrm{C}$ until assessed.

\section{Tear sample preparation}

A detailed description of the sample preparation steps has been previously described $[17,18]$. In brief, samples were flushed from capillaries with $0.5 \%$ sodium dodecyl sulphate (SDS) and protein concentration was measured with DC protein assay kit (Bio-Rad laboratories Inc, Hercules, USA). 15/210 samples did not have sufficient amounts of proteins ( $\geq 5 \mu \mathrm{g})$ for further analysis. Acetone-precipitated proteins were dissolved in 2\% SDS, reduced by tris-(2-carboxyethyl)phosphine (TCEP) and alkylated with iodoacetamide (IAA) on $30 \mathrm{kDa}$ molecular weight cut-off filters (Pall Corporation, Port Washington, NY, USA) in order to lower the sample complexity and remove small contaminants. Samples were digested with trypsin (Sciex, Framingham, USA) and cleaned and desalted with C18 tips (Thermo Fisher Scientific). Unless otherwise stated all reagents were purchased from Sigma-Aldrich (St. Louis, MO, USA).

\section{Identification and SWATH-MS quantification of tear proteins}

The samples were analysed using the Eksigent 425 NanoLC coupled to the high speed TripleTOF ${ }^{\mathrm{TM}}$ $5600+$ mass spectrometer (Ab Sciex, Concord, Canada). Equal amounts $(3 \mu \mathrm{g})$ of tear peptide mix from each sample were first loaded into the trap column (ChromXP C18-CL $3 \mu \mathrm{m} 120 \AA, 200 \mu \mathrm{m} \times 0.5 \mathrm{~mm})$ and then separated using a cHiPLC column (ChromXP C18-CL $3 \mu \mathrm{m}$ $120 \AA$, $75 \mu \mathrm{m} \times 15 \mathrm{~cm}$ ). Peptides were introduced into the mass spectrometer (MS) via NanoSpray III source and analysed with 120 min 6 step gradients using eluent $\mathrm{A}(0.1 \% \mathrm{FA}$ in $1 \% \mathrm{ACN})$ and eluent $\mathrm{B}(0.1 \% \mathrm{FA}$ in $\mathrm{ACN}$ ) at $300 \mathrm{nl} / \mathrm{min}$. The spectral library for the SWATH analysis was created by the data dependent-acquisition (DDA) method using the ProteinPilot ${ }^{\circledR}$ software version 4.7 (Ab Sciex, Concord, Canada) and false discovery rate $(\mathrm{FDR})<1 \%$. For protein identification, MS/MS spectra were searched against the UniProtKB/Swiss-Prot human database (version 2016_01). Protein quantification against the obtained spectral library was performed using PeakView ${ }^{\circledR}$ and MarkerView ${ }^{\circledR}$ software (Sciex, Redwood City, USA). Equal amounts of proteins were used, and two replicate MS-analyses were performed from each sample. Due to instrument errors (column or trap column break down) and small sample size (not enough sample for additional injections) 3 samples were lost. Ten peptides from albumin were chosen for the retention time calibration for all samples. Five transitions per peptide and 1-15 peptides were used for the peak area calculations. The most relevant/significant differentially expressed proteins were subjected to manual inspection of peptides, including correct peak selection check (FDR $1 \%$, 99\% peptide confidence level) and chromatogram inspection in relation to library chromatogram. Protein quantification results are presented as a combination of protein specific peptides peak intensities from SWATHMS measurement and here referred to as protein expression. The key parameters used in MS analysis and protein identification have been described in detail before [17, $18]$.

\section{Statistical analysis}

Only one eye from each patient was included in the analysis of the proteomics data. The quantified proteomics data was $\log _{2}$-transformed and the quantile normalization was applied to reduce any potential bias. The intraclass correlation coefficient (ICC) was used to establish the quality of the MS analysis replicates. The means were taken from the MS analysis replicates. The differential expression analyses between visits, i.e. preoperative (Vpre), postoperative (Vpost) and one average one month after the operation $(\mathrm{V} 1 \mathrm{~m})$, were performed using the Wilcoxon signed rank test with the continuity correction to account for the repeated measures.

The Spearman's rank correlation was implemented to measure correlation between continuous clinical variables (flap thickness and correction (target refraction-preoperative spherical equivalent refraction $(\mathrm{SEQ})))$ and protein expression changes between visits.

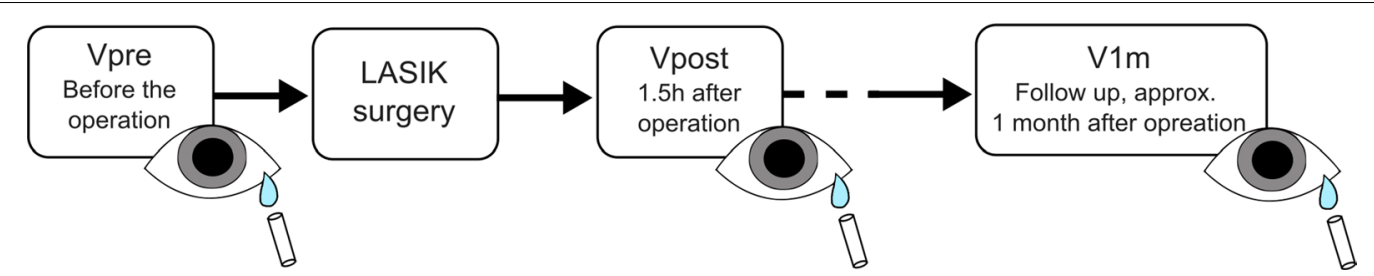

Fig. 1 Study outline. The initial capillary sample was taken before the operation (Vpre) and the postoperative sample (Vpost) was taken $1.5 \mathrm{~h}$ after the LASIK surgery. The final follow-up visit and sampling took place approximately 1 month after the surgery $(\mathrm{V} 1 \mathrm{~m})$ 
The correlation coefficients were estimated for myopic patients only, due to differences in surgical strategies and a low number of hyperopic patients. In addition, protein expression level differences between sexes, flap diameter $(<9.4 \mathrm{~mm}$ vs $\geq 9.4 \mathrm{~mm})$ and hinge length $(<4 \mathrm{~mm}$ vs $\geq 4 \mathrm{~mm}$ ) were evaluated with Wilcoxon rank sum test. Flap diameter and hinge length variables were discretized into two groups since both variables were restricted to a small number of possible values.

The multiple testing was accounted for by implementing the Benjamini-Hochberg adjustment to the raw p-values. A significance level (alpha) of 0.05 was used as a threshold in all tests, excluding the Spearman's correlation, where a threshold on absolute correlation coefficient (Rho) of 0.5 was implemented. The $\mathrm{R}$ software version 3.2.3 [19] was used to perform the analyses. Online database tool STRING [20] was used to identify proteinprotein interaction networks (the minimum required interaction score set as "high confidence") and the IPA (Ingenuity Pathway Analysis) software (IPA, QIAGEN Redwood City, USA) was used to identify enriched biological functions.

\section{Results}

\section{Patient demography}

In total, 70 patients were included in the study. The mean age of the patients was $38.4 \pm 10.3$ years and ranged from 18 to 57 years. The data consisted of 42 females and 28 males and the mean ages of the two groups did not differ significantly $(P=0.79)$. Refractive corrections were performed for both myopic and hyperopic subjects (59 and 11 patients, respectively) and hence, these two groups are shown separately in Table 1, which summarizes further the clinical information.

\section{Proteomics data and differential expression analysis}

With a spectral library of 1329 proteins, 747 proteins had distinct peptides and were quantified in all samples. The SWATH-MS was able to produce reproducible results with a mean ICC of 0.97 between MS analysis replicates. Number of peptides used in the quantitation of proteins is listed in Additional file 1.

The comparison of protein expression levels between Vpre and Vpost time points identified altogether 158 differentially expressed proteins (median fold change $(\mathrm{FC})>1.5$ or $<0.67$, adjusted $p$ value $<0.05$ ). Out of these proteins, 81 were upregulated and 77 were downregulated. Most notably, the downregulated protein list included 49 immunoglobulin subunits. These are colored in Fig. 2a, where some other individual proteins are also labeled. The full results can be found from the supplementary materials (Additional file 2).
Table 1 Clinical information of the patients

\begin{tabular}{|c|c|c|c|c|}
\hline \multirow[t]{2}{*}{ Clinical variable } & \multicolumn{2}{|c|}{ Myopic $(n=59)$} & \multicolumn{2}{|c|}{ Hyperopic $(n=11)$} \\
\hline & Vpre & V1m & Vpre & V1m \\
\hline SEQ (D) & $-3.9 \pm 2$ & $-0.2 \pm 0.7$ & $2 \pm 1.1$ & $-1 \pm 0.9$ \\
\hline $\begin{array}{l}\text { Corneal thickness } \\
(\mu \mathrm{m})\end{array}$ & $540.9 \pm 33.8$ & $479.2 \pm 44.4$ & $538 \pm 25.9$ & $530.5 \pm 24.6$ \\
\hline $\mathrm{IOP}(\mathrm{mmHg})$ & $16.5 \pm 2.8$ & $11.7 \pm 1.9$ & $16.3 \pm 2.7$ & $13.8 \pm 3.2$ \\
\hline Correction (D) & $3.5 \pm 1.9$ & & $3 \pm 1.1$ & \\
\hline $\begin{array}{l}\text { Flap diameter } \\
(\mathrm{mm})\end{array}$ & $9.3 \pm 0.1$ & & $9.4 \pm 0.1$ & \\
\hline $\begin{array}{l}\text { Flap thickness } \\
\qquad(\mu \mathrm{m})\end{array}$ & $93.8 \pm 6.9$ & & $89.5 \pm 5$ & \\
\hline Predictability (D) & $0.2 \pm 0.3$ & & $0.1 \pm 0.4$ & \\
\hline
\end{tabular}

The values are shown as mean \pm standard deviation

SEQ spherical equivalent refraction, $I O P$ intraocular pressure, Correction abs(target refraction-preoperative SEQ), Predictability abs(postoperative SEQ - target refraction), Vpre preoperative visit, Vpost postoperative visit $1.5 \mathrm{~h}$ after surgery, $V 1 m$ follow-up visit 1 month after surgery

A similar differential expression comparison was performed for Vpost and V1m time points, and altogether 110 statistically significant proteins were identified to have median $\mathrm{FC}>1.5$ or $<0.67$ and adjusted $p$ value $<0.05$. Fifty-six proteins were upregulated and 54 were downregulated (Fig. 2b) and full results are listed in Additional file 3 . No statistically significant differences were present in comparisons between Vpre and V1m time points.

As shown in Fig. 2c, there were several proteins, which had changed expression levels in both time points (104 proteins) and it is worth noting, that all these proteins had the opposite direction between the visit comparisons, i.e., the protein expression level changes returned back to preoperative values 1 month after surgery thus indicating recovery after the surgical trauma. This group of proteins included, e.g., albumin (ALB), annexins A1 and A2 (ANXA1, ANXA2), alpha-enolase (ENO1), 14-3-3 protein zeta/delta (YWHAZ) and several S100A proteins (S100A4, S100A6, S100A9), which were initially increased $1.5 \mathrm{~h}$ after the surgery, and were then decreased by the final visit 1 month after surgery. Some proteins, such as 14-3-3 protein beta/alpha (YWHAB) as well as 47 out of the 49 immunoglobulin subunits were downregulated $1.5 \mathrm{~h}$ post-surgery and returned to normal within 1 month. Figure 3 displays the protein-protein interactions of these common proteins, found from both comparisons.

In addition to these proteins, some proteins were significantly changed only between the Vpre and Vpost visits (54) or had altered expression only 1 month after the surgery (6), as shown in Fig. 2c. The most notably changed proteins, i.e. those with significant changes according to one time point comparison $(\mathrm{FC}>1.5$ or $<0.67$ and 

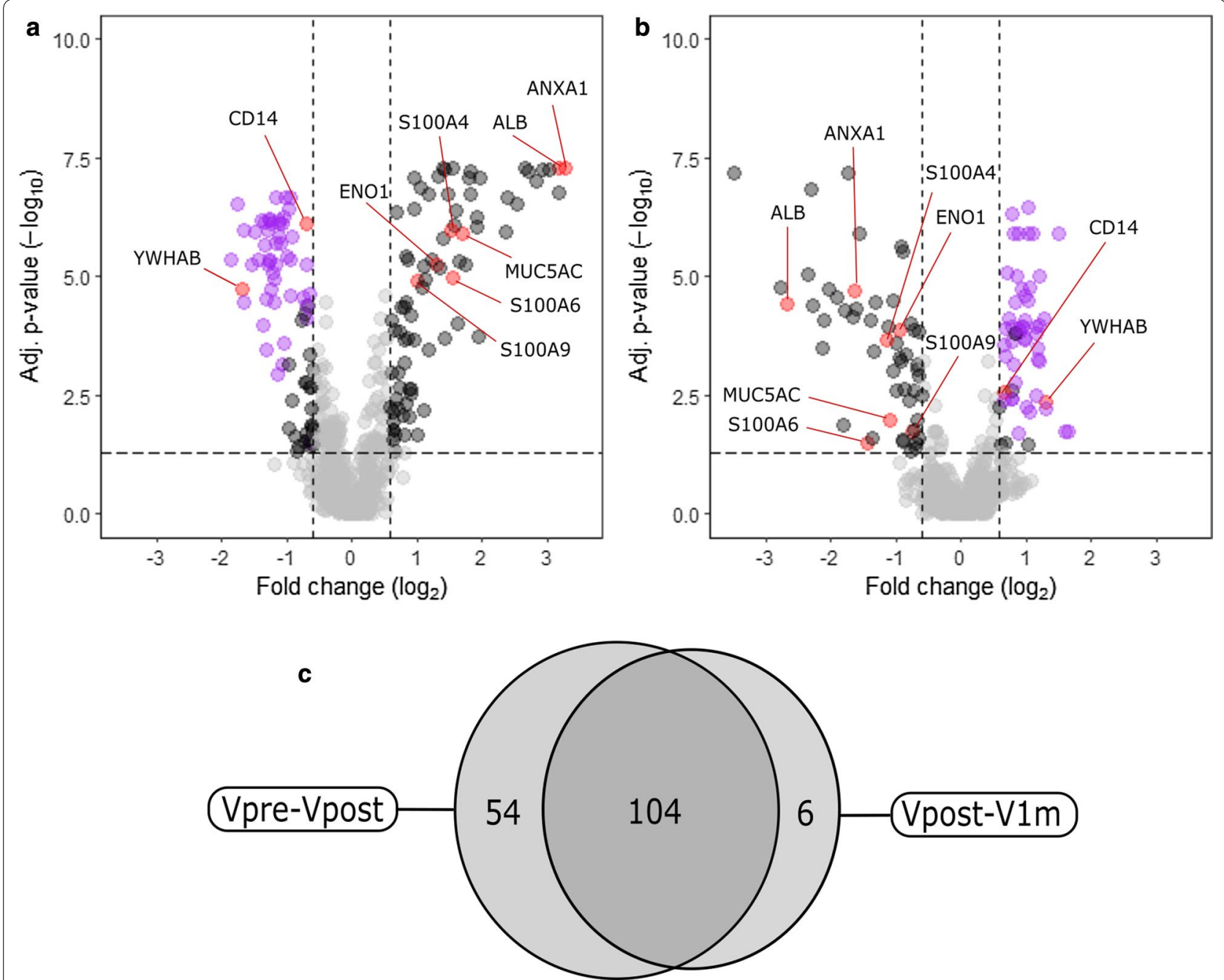

Fig. 2 Differentially expressed proteins between pre- and postoperative visits' tear samples. $\mathbf{a}, \mathbf{b}$ The volcano plots display the results of differential expression analyses for $\mathbf{a}$ Vpre-Vpost ( 55 sample pairs available) and $\mathbf{b}$ Vpost-V1 $\mathrm{m}$ (35 sample pairs available) comparisons. The adjusted p-values (y-axis) are shown in - $\log _{10}$ scale and the dashed horizontal line represents the adjusted $p$-value threshold of 0.05 and the points above this horizontal line are statistically significant. The median fold changes are in the $x$-axis and are in $\log _{2}$ scale. The two dashed lines here are showing the 1.5 and 0.67 -fold change thresholds. The values on the left side of the thresholds represent proteins, which were downregulated and the points on the far right were upregulated. Some of the proteins of interest are named (red) and the purple dots represent immunoglobulin subunits. $\mathbf{c}$

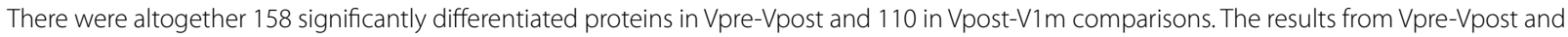
Vpost-V1m comparisons share altogether 104 proteins, which are differentially expressed in both comparisons

$p$ value $<0.05)$ and no notable change in the other time point comparison $(\mathrm{FC}<1.25$ or $>0.8$ and $p$ value $>0.05)$, included some well-known tear fluid proteins visualized in Fig. 4.

\section{Pathway analysis of the differentially expressed proteins}

The pathway analysis was conducted using the IPA and the results obtained from the separate Vpre-Vpost and the Vpost-V1m comparisons are summarized in Table 2. From the enrichment results relating to diseases and biological functions, it is evident that $1.5 \mathrm{~h}$ after the surgery there was a rapid activation of pathways related to cell movement of immune cells, e.g. myeloid cells, phagocytes and leukocytes, inflammation, angiogenesis and viral infection. In addition, the pathways related to organismal survival were decreased $1.5 \mathrm{~h}$ after the surgery. These actions were then reversed by the final visit, 1 month after the surgery, where the cell movement and viral infection were decreased, and organismal survival and cell death were increased. An increase in vasculature development and energy production only take place when comparing the Vpre and Vpost time points. 


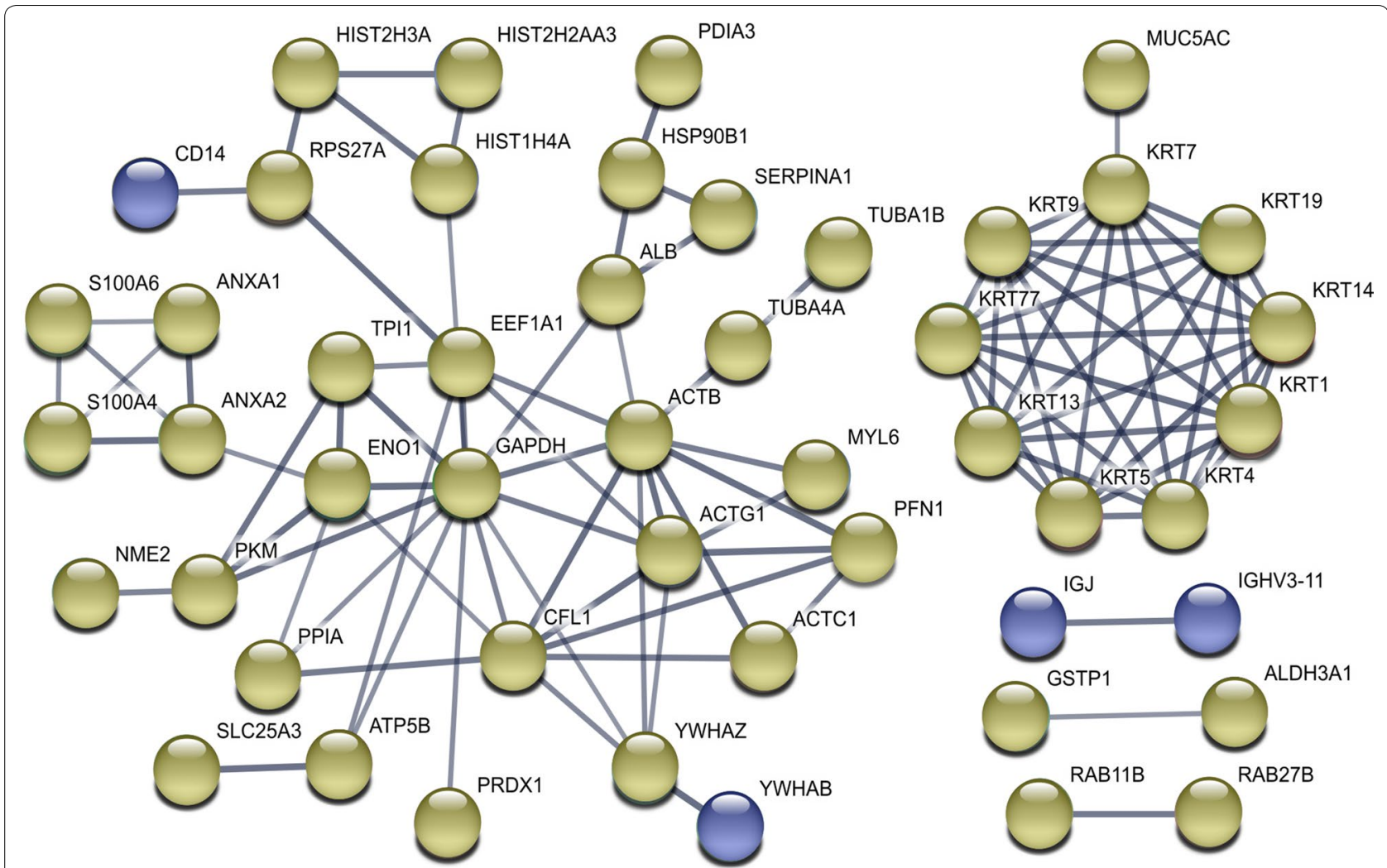

$\Delta$ Vpost, $\nabla$ V1m

$\boldsymbol{\nabla}$ post, $\boldsymbol{\Delta} \mathrm{V} 1 \mathrm{~m}$

Fig. 3 Proteins differing significantly in both time point comparisons (Vpre-Vpost and Vpost-V1m). The protein-protein interaction network displays proteins, which had increased expression levels $1.5 \mathrm{~h}$ after the surgery and decreased expression levels 1 month after the surgery (in yellow) and proteins, which were initially decreased $1.5 \mathrm{~h}$ post-surgery and increased 1 month after surgery (in blue). Only proteins, which could be identified in STRING and had protein-protein connections are included
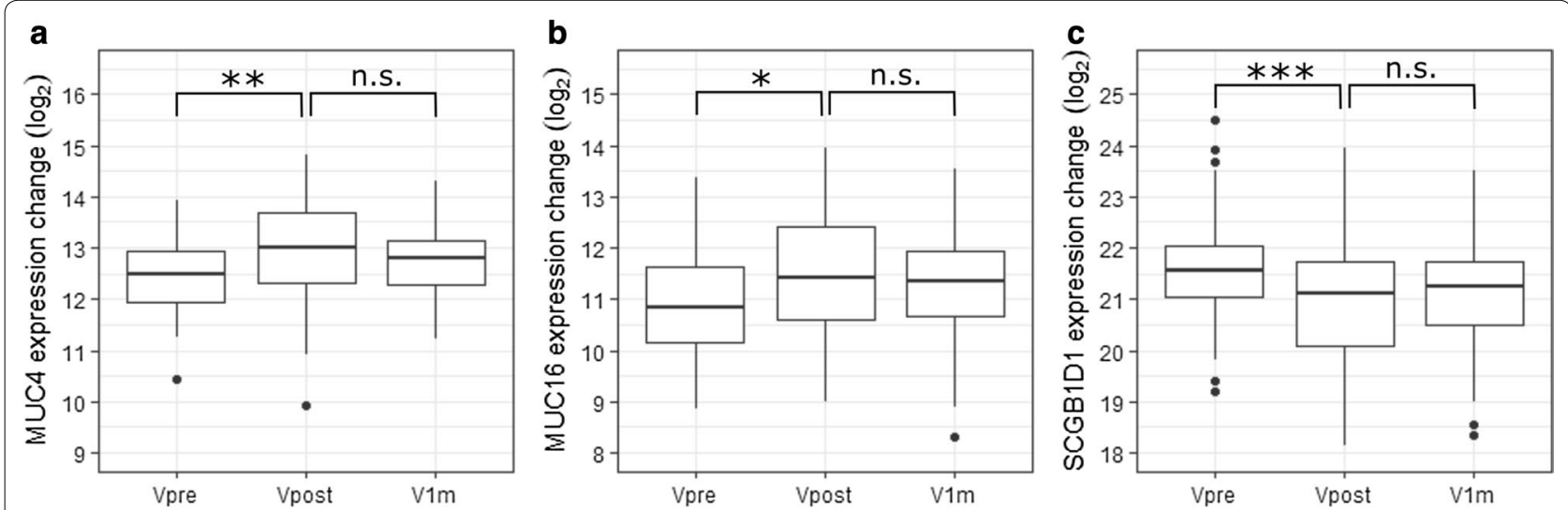

Fig. 4 Mucins (MUC4 and MUC16) and secretoglobin family 1D member 1 (SCGB1D1) had significant expression level changes only between Vpre and Vpost time points. a, b Mucins MUC4 and MUC16 had increased expression levels $1.5 \mathrm{~h}$ after the surgery (Vpre-Vpost) and these levels did not reduce between the postoperative visits (Vpost-V1m). c Notably decreased expression levels of SCGB1D1 were observed between Vpre and Vpost visits, while no difference was again observed between Vpost and $\mathrm{V} 1 \mathrm{~m} .{ }^{*} P<0.05$; ${ }^{* *} P<0.01$; ${ }^{* *} P<0.001$; n.s. non-significant 
Table 2 The pathways associated with surgery effects and recovery

\begin{tabular}{|c|c|c|c|c|c|c|c|}
\hline \multirow[t]{2}{*}{ Categories } & \multirow[t]{2}{*}{ Diseases or Functions Annotation } & \multicolumn{3}{|c|}{ Vpre vs Vpost } & \multicolumn{3}{|c|}{ Vpost vs V1m } \\
\hline & & Z-score & $\operatorname{Adj} P$ & Protein count & Z-score & Adj $P$ & Protein count \\
\hline \multirow[t]{2}{*}{ Organismal survival } & Morbidity or mortality & -4.3 & 0.007 & 41 & 3.5 & 0.021 & 29 \\
\hline & Organismal death & -4.2 & 0.009 & 40 & 3.3 & 0.028 & 28 \\
\hline Cell death and survival & Apoptosis & & & & 2.1 & 0.003 & 34 \\
\hline Protein synthesis & Quantity of enzyme & -2.2 & 0.025 & 5 & & & \\
\hline \multirow[t]{2}{*}{ Tissue morphology } & Quantity of leukocytes & -2.4 & 0.005 & 21 & & & \\
\hline & Quantity of blood cells & -2.1 & 0.007 & 22 & & & \\
\hline Inflammatory response & Inflammation of organ & -2.1 & $<0.001$ & 38 & & & \\
\hline Energy production & Concentration of ATP & 2.1 & 0.039 & 5 & & & \\
\hline Inflammatory response & Inflammatory response & 2.3 & 0.006 & 19 & & & \\
\hline \multirow[t]{10}{*}{ Cellular movement } & Chemotaxis & 2.6 & 0.020 & 13 & & & \\
\hline & Chemotaxis of leukocytes & 2.6 & 0.003 & 12 & & & \\
\hline & Chemotaxis of myeloid cells & 2.6 & 0.005 & 10 & & & \\
\hline & Chemotaxis of neutrophils & 2.2 & 0.022 & 6 & & & \\
\hline & Chemotaxis of phagocytes & 2.6 & 0.006 & 10 & & & \\
\hline & Migration of cells & 2.9 & $<0.001$ & 71 & -2.6 & $<0.001$ & 56 \\
\hline & Leukocyte migration & 2.3 & $<0.001$ & 54 & & & \\
\hline & Cell movement & 3.1 & $<0.001$ & 79 & -3.0 & $<0.001$ & 62 \\
\hline & Cell movement of leukocytes & 2.4 & 0.006 & 19 & & & \\
\hline & Cell movement of myeloid cells & 2.5 & 0.007 & 15 & & & \\
\hline \multirow{2}{*}{$\begin{array}{l}\text { Vascular system develop- } \\
\text { ment and function }\end{array}$} & Angiogenesis & 3.3 & 0.024 & 19 & & & \\
\hline & Development of vasculature & 3.3 & 0.036 & 20 & & & \\
\hline \multirow[t]{4}{*}{ Infectious diseases } & Viral Infection & 3.6 & $<0.001$ & 37 & -2.8 & 0.002 & 24 \\
\hline & Replication of virus & 3.2 & 0.003 & 15 & -2.6 & 0.010 & 11 \\
\hline & Replication of RNA virus & 2.9 & 0.007 & 13 & -2.2 & 0.028 & 9 \\
\hline & Infection by RNA virus & & & & -2.6 & 0.028 & 13 \\
\hline
\end{tabular}

Cancer-related terms are excluded from the table

Vpre preoperative visit, Vpost postoperative visit $1.5 \mathrm{~h}$ after surgery, $\mathrm{V} 1 \mathrm{~m}$ follow-up visit $1 \mathrm{month}$ after surgery

\section{Relationship between clinical variables and the proteomics in myopic eyes}

Clinical variables, which are known to be connected to post-LASIK dry eye, i.e. sex as well as some surgical parameters such as the amount of correction, flap thickness and flap diameter were also evaluated in connection to the proteomics data. As seen from Fig. 5, several proteins correlated with the amount of correction and flap thickness. More specifically, retinoic acid receptor responder protein 1 (RARRES1), protein disulfideisomerase A4 (PDIA4) and apolipoprotein B-100 (APOB) expression levels were noted to be more decreased 1 month after surgery for those patients, who had a greater attempted correction (Fig. 5a-c). Flap thickness also appeared to influence type II cytoskeletal 6A keratin (KRT6A) expression level changes 1 month after surgery in comparison to preoperative values (Fig. $5 \mathrm{~d}$ ).

In addition to examining the correlations between clinical variable and protein expression level changes, we also examined the correlations of clinical variables and protein expression levels in individual time points. According to our results, V1m expression levels of PDIA4 and phosphatidylethanolamine-binding protein 1 (PEBP1) were correlating with the amount of correction (Fig. 5e, f). Further results of the correlation comparisons are listed in Additional file 4.

Flap diameter was also examined in connection to protein expression levels, and a flap diameter of $9.4 \mathrm{~mm}$ or over appeared to influence the expression levels of polymeric immunoglobulin receptor (PIGR) and immunoglobulin heavy variable 3-23 (IGHV3-23). Both proteins were decreased significantly 1 month after surgery for patients with a larger flap diameter (Fig. $5 \mathrm{~g}, \mathrm{~h}$ ). Hinge length and sex were not affecting the protein expression levels according to our results.

\section{Discussion}

It is known that corneal keratorefractive surgery affects both epithelial and stromal layers of the cornea and initiates a complex corneal wound healing cascade 

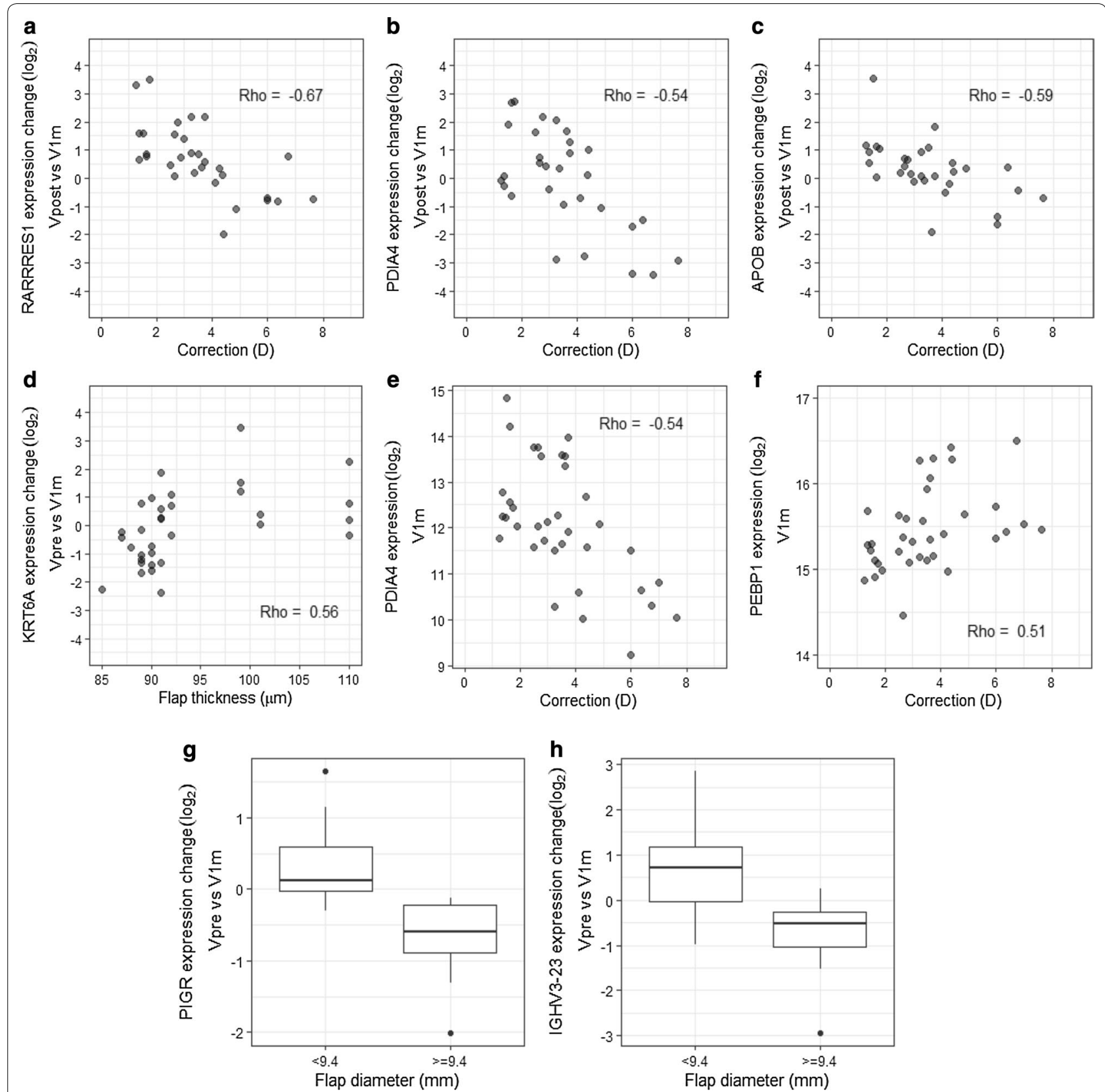

Fig. 5 The amount of correction, flap thickness and flap diameter affect the protein expression levels. a-c Retinoic acid receptor responder protein 1 (RARRES1), protein disulfide-isomerase A4 (PDIA4), and apolipoprotein B-100 (APOB) expression level changes between Vpost and $\mathrm{V} 1 \mathrm{~m}$ time points correlated negatively with the amount of correction. $\mathbf{d}$ Flap thickness correlated positively with type II cytoskeletal 6A keratin (KRT6A) expression level changes between Vpre and $\mathrm{V} 1 \mathrm{~m}$. e, f $\mathrm{V} 1 \mathrm{~m}$ expression levels of PDIA4 had a negative correlation and phosphatidylethanolamine-binding protein 1 (PEBP1) a positive correlation against the correction amount. $\mathbf{g}, \mathbf{h}$ Flap diameter of $9.4 \mathrm{~mm}$ or over was associated with a decrease in expression levels of polymeric immunoglobulin receptor (PIGR) and immunoglobulin heavy variable 3-23 (IGHV3-23) 1 month after the surgery

immediately after the surgery [9]. In the outermost epithelial layer, after an initial latent phase, high levels of adenosine triphosphate (ATP) are released and epithelial cells migrate to the edges of the wound in the early stages of wound healing [21, 22]. In the underlying corneal stroma, the wound healing is initiated by a rapid release of cytokines from the epithelium and lacrimal gland, causing stromal keratocyte apoptosis [10, 23, 24], followed by the remaining keratocytes' proliferation to fibroblasts and myofibroblasts (reviewed by Wilson et al. 
[11]). In addition, migration of immune and inflammatory cells takes place within the first hours after corneal epithelial injury [25].

Our proteomics results indicated that tear fluid, from its part, enhances these biological processes early on, as we could identify a large collection of tear proteins, which had significantly altered expression levels $1.5 \mathrm{~h}$ after LASIK surgery. The pathway analyses further confirmed that these initial protein profile changes were connected to increased cell migration of immune cells, inflammatory response as well as concentration of ATP, which is required to fuel the increased energy consumption needed in all phases of the wound healing. In addition, the findings suggested increased activity in pathways connected to viral infection and angiogenesis. Despite the activation of angiogenic pathways in our results, the antiangiogenic signals are likely to balance these in order to maintain the avascularity, which is crucial for the normal function of cornea. Regarding the increased viral infection activity, there has been evidence that some, albeit a very small proportion of subjects, experience an activation of herpes simplex virus (HSV) keratitis after LASIK surgery [26, 27]. However, from the perspective of proteomics, this matter warrants more investigation.

The initial corneal wound healing effects, i.e. cell migration as well as keratocyte apoptosis and activation, peak within hours or days after surgery $[25,28]$, however, regeneration of nerve fibres, restoration of epithelial basement membrane and stromal remodelling may take from several months to years [29-31]. According to our tear proteomics results, approximately twothirds of the proteins (104/158), which were identified to have altered expression levels $1.5 \mathrm{~h}$ after the surgery, had an expression level change of opposite direction 1 month after the surgery, while a third of the proteins did not display a similar return to normal. The proteins, which were observed to return to normal within the first month included various well-known tear fluid proteins. For example, ALB, ANXA1, ANXA2, ENO1, S100A4, S100A6, S100A9, actin, cytoplasmic 1 (ACTB), alpha1-antitrypsin (SERPINA1), aldehyde dehydrogenase, dimeric NADP-preferring (ALDH3A1) and glutathione S-transferase P (GSTP1) were initially upregulated after the LASIK surgery, but expression levels had decreased back by the 1 month visit. According to previous studies, the expression levels of these proteins have been observed to be increased in aqueous or Sjögren syndrome dry eye, or both [32-36], thus being possible tear fluid indicators of ocular surface stress and possible inflammation. Our results, showing these proteins return to normal, therefore indicate that the acute state in the tear film has passed within the first postoperative month.
In addition to the inflammation-associated proteins, several keratins (KRT1, KRT4, KRT5, KRT7, KRT9, KRT13, KRT14, KRT19, KRT77), which are known to be vital structural proteins of epithelial cells, were increased initially. The observed expression levels indicate that the keratins are released to the tear fluid as a result of the wounding, which is expected. The expression levels of keratins were then reduced back to preoperative levels during the first postoperative month according to our results.

The results also included a collection of proteins, which initially had decreased protein expression levels $1.5 \mathrm{~h}$ after surgery, and which were then increased to preoperative levels within the first postoperative month. This protein group consisted almost entirely of immunoglobulin subunits, or associated proteins, and interestingly, previous studies have linked increased immunoglobulin expression levels with various ocular surface diseases and prolonged contact lens wear $[37,38]$. The reasons for decreased levels of immunoglobulins in acute stress in our results remain unclear. One hypothesis could be that the tear fluid protein profiles $1.5 \mathrm{~h}$ after surgery could also be connected to surgery-associated eye drops, therefore affecting proteins connected to immune response and their expression levels. Alternatively, in the initial stages of the wound healing, i.e., hours after the surgery, the immunoglobulins may be bound to the wounded tissue or their production could take longer, thus causing them not being observed in the tear fluid.

According to our results, a third of the proteins with initially altered protein expression levels did not return to preoperative levels within 1 month. However, it is worth noting that in many cases, the fold changes indicated a shift back to preoperative levels, but the p-values were above the threshold, likely to due to large variation. Therefore, we decided to focus on proteins, which had a strong altered expression level (FC $>1.5$ or $<0.67$ and $p$ value $<0.05)$ in one comparison and no notable change in the other ( $\mathrm{FC}<1.25$ and $>0.8$ and $p$ value $>0.05)$. This way, after careful peak checking three well-known ocular surface proteins were studied further. Two of the proteins were membrane mucins, MUC4 and MUC16, which had increased expression levels $1.5 \mathrm{~h}$ after surgery and were not displaying signs of recovery, i.e., decrease, at 1 month after surgery. Increased expression levels of mucins in tear fluid, often connected to epithelium barrier function, could indicate an increased shedding from the epithelium, possibly due to pro-inflammatory agents in the tear films, such as tumor necrosis factor (TNF) [39]. This would suggest that from the perspective of epithelium layer, some wound healing functions are still ongoing 1 month after surgery. In addition to mucins, we found that androgen-associated secretoglobin family $1 \mathrm{D}$ 
member 1 (SCGB1D1) had significantly decreased protein expression level changes $1.5 \mathrm{~h}$ after the surgery and no notable change from these levels 1 month after the surgery. Decreased expression levels of SCGB1D1, as well as other proteins belonging to the secretoglobin family, have previously been connected to Meibomian gland dysfunction and evaporative dry eye [40, 41]. Overall, it would appear, that a small number of tear fluid proteins does not return to preoperative levels within 1 month, and it could be of interest to study these proteins further in relation to the development of adverse effects and more efficacious post-LASIK treatment methods.

In addition to differences between time points, the correlations between various clinical variables and protein expression levels were also evaluated. According to our results, particularly the amount of correction had an effect on the expression levels and expression level changes of some proteins. RARRES1 and APOB, both connected to peroxisome proliferating activating receptor (PPAR) and fatty acid metabolism [42, 43], had greater decreases in expression levels 1 month after surgery in connection to larger amount of correction. Interestingly, since the different PPAR isoforms have been connected to inflammation and wound healing of cornea, their antagonists have been suggested as potential therapeutic targets in corneal wound healing [44-46]. The potential roles of RARRES1 and APOB to the corneal wound healing should therefore be further studied. In addition, PDIA4 correlated similarly with the amount of correction. PDIA4 is closely connected to platelet activation as well as fibrin formation and thrombosis [47], however, this protein's connection to the healing process of avascular cornea, are yet unknown. According to these correlation results, a greater amount of correction is connected to a decrease of several proteins closely connected to wound healing and associated inflammation, possibly indicating differing wound healing response caused by the deeper stromal excimer laser ablation in greater corrections. This may reflect differences in density and function of keratocytes and nerve fibers in different stromal layers.

Flap parameters were also associated to the expression level changes of proteins. Flap diameter affected the expression level changes of PIGR and IGHV3-23, both molecules connected to immunoglobulins. More specifically, when the flap diameter was $9.4 \mathrm{~mm}$ or larger, there was a notable decrease in expression levels 1 month after surgery, while with smaller diameters, the expression levels did not change or increased. As discussed earlier, our results showed that $1.5 \mathrm{~h}$ after LASIK surgery, many immunoglobulins were initially decreased, followed by an increase back to normal within 1 month. This additional observation indicating that not all proteins return to preoperative levels for some subjects with a larger flap diameter, could suggest that larger flap diameter, which could be connected to wider corneal nerve damage, does have an effect on the ocular surface recovery.

The LASIK-induced effects in the tear fluid have been studied previously to some extent, mainly through various cytokine studies $[12,13,15,16]$, but only one previous publication has evaluated the LASIK's effects on larger protein profile [14]. In the study by D'Souza et al., the focus was on comparison of two types of femtosecond lasers, Visumax and Intralase, at later time points ( 1 week and 3 months) and thus, the initial changes taking place in the tear film were not examined. The results obtained from the D'Souza's study and our study are not fully comparable also due to different sample collection methods (Schirmer's strip vs capillary) and mass spectrometry methodology (iTRAQ vs SWATH-MS), which can both affect the protein identification and thus, protein profile results $[18,48]$. Therefore, to our knowledge, our study is the first discovery proteomics study focusing on the initial functional changes occurring in the tear film after a LASIK surgery.

In surgery, medication is a vital and unavoidable part of the operation and always prone to influence the biological processes taking place during and after the surgery. In this study, the perioperative levofloxacin, diclofenac, brimonidine tartrate, oxybuprocaine hydrochloride and postoperative dexamethasone and chloramphenicol are likely to have at least short-term effects on the biological functions of ocular surface as well as the tear fluid composition, thus potentially affecting the postoperative samples' protein profiles. However, as the drugs are a vital and standardized part of the LASIK surgery and as the ocular surface during and after LASIK surgery is not comparable to a normal, healthy ocular surface, examination of the pharmaceutical treatments' effects alone is not plausible. The pre-, peri- and postoperative topical drugs are therefore important and necessary factors when evaluating the tear protein profile changes and altered biological functions after LASIK.

Overall, our findings help us understand better the initial biological changes taking place in the ocular surface after LASIK surgery. In the future, both the biological functions and individual proteins identified in our study could benefit the development of methods used to follow the post-LASIK healing process and to identify subjects, who do not recover as expected. In addition, knowing the changes taking place in tear protein profiles after a successful LASIK operation, could help develop new treatment approaches to prevent or reduce LASIK-related adverse effects. 


\section{Conclusions}

The present study showed that an uneventful femtosecond LASIK refractive surgery induced significant migration and inflammation-associated changes in tear proteomic profile quickly after the operation, but the expression of majority of proteins recovered almost completely to the preoperative levels during the first month. A small subset of proteins did not display similar return to normal, demonstrating that some wound healing functions remain ongoing also in the tear fluid 1 month after the operation. We also identified several proteins, which correlated in myopic eyes with the amount of refractive correction, flap thickness and flap diameter, indicating that the surgical variables affect the long-term, individual wound healing responses. The individual proteins identified in our study are potential targets for the followup and modification of LASIK-induced biochemical processes.

\section{Supplementary information}

Supplementary information accompanies this paper at https://doi. org/10.1186/s12014-020-09303-9.

Additional file 1. Number of peptides used in the quantitation of proteins.

Additional file 2. Proteins with significantly changed expression levels (adj. P-value <0.05) between Vpre and Vpost time points.

Additional file 3. Proteins with significantly changed expression levels (adj. P-value $<0.05$ ) between Vpost and V1 $\mathrm{m}$ time points.

Additional file 4. Correlations between clinical variables and protein expression levels performed with Spearman's correlation. Listed are only proteins with Rho $>0.5$ or $<-0.5$.

\section{Abbreviations}

ACTB: Actin, cytoplasmic 1; ALB: Albumin; ALDH3A1: Aldehyde dehydrogenase, dimeric NADP-preferring; ANXA1: Annexin 1; ANXA2: Annexin 2; APOB: Apolipoprotein B-100; ATP: Adenosine triphosphate; CDVA: Corrected distance visual acuity; DDA: Data dependent-acquisition; ECM: Extracellular matrix; ELISA: Enzyme-linked immunosorbent assay; ENO1: Alpha-enolase; FC: Fold change; FDR: False discovery rate; GSTP1: Glutathione S-transferase P; HSV: Herpes simplex virus; IAA: Iodoacetamide; ICC: Intraclass correlation; IGHV3-23: Immunoglobulin heavy variable 3-23; IOP: Intraocular pressure; IPA: Ingenuity Pathway Analysis; iTRAQ: Isobaric tags for relative and absolute quantitation; KRT6A: Type II cytoskeletal 6A keratin; LASIK: Laser in situ keratomileusis; PDIA4: Protein disulfide isomerase A4; PEBP1: Phosphatidylethanolamine-binding protein 1; PIGR: Polymeric immunoglobulin receptor; PPAR: Peroxisome proliferating activating receptor; RARRES1: Retinoic acid receptor responder protein 1; SCGB1D1: Secretoglobin family 1D member 1; SDS: Sodium dodecyl sulphate; SEQ: Spherical equivalent refraction; SERPINA1: Alpha-1-antitrypsin; SWATH-MS: Sequential window acquisition of all theoretical fragment ion spectra mass spectrometry; TCEP: Tris-(2-carboxyethyl)phosphine; TNF: Tumor necrosis factor; UDVA: Uncorrected distance visual acuity; YWHAB: 14-3-3 Protein beta/alpha; YWHAZ: 14-3-3 Protein zeta/delta.

\section{Acknowledgements}

Saara Lähdekorpi, Antti Jylhä, Teppo Rajala and Vesa Alho are thanked for their skillful technical assistance.

\section{Authors' contributions}

PM, UA, JP and HU contributed to the design of the work. PM and JP performed the surgical procedures. JN executed the data acquisition, data analysis and visualizations. JN, PM, UA and HU interpreted the analysis results. All authors contributed to the manuscript preparation. Mass spectrometry instruments and expertise in proteomics were provided by the Department of Ophthalmology and its technical staff. All authors read and approved the final manuscript.

\section{Funding}

This work was supported by Finnish Funding Agency for Technology and Innovation (TEKES, Finland, grant number: 40087/12) and Elsemay Björn Fund (JN, UA, HU). The funding sources were not involved in study design, data collection, analysis and interpretation, decision to publish, or writing of this manuscript.

\section{Availability of data and materials}

The datasets used and analysed during the current study are available from the corresponding author on reasonable request.

\section{Ethics approval and consent to participate}

The present study was approved by the Ethical Committee of Pirkanmaa Hospital District (R13074), Finland, and performed according to The Declaration of Helsinki. An informed consent was obtained from all patients.

\section{Consent for publication}

Not applicable.

\section{Competing interests}

JN, PM, UA, LO and HU declare that they have no competing interests. JP has financial interest in the Ziemer Ophthalmic Systems. The authors report no other conflicts of interest in this work.

\section{Author details}

1 SILK, Department of Ophthalmology, Faculty of Medicine and Health Technology, Tampere University, PL 100, 33014 Tampere, Finland. ${ }^{2}$ Silmäasema Eye Hospital, Tampere, Finland. ${ }^{3}$ TAUH Eye Center, Tampere University Hospital, Tampere, Finland.

Received: 18 June 2020 Accepted: 13 October 2020

Published online: 19 October 2020

\section{References}

1. Solomon KD, de Castro LE, Sandoval HP, Biber JM, Groat B, Neff KD, et al. LASIK world literature review: quality of life and patient satisfaction. Ophthalmology. 2009;116(4):691-701.

2. Donnenfeld ED, Solomon K, Perry HD, Doshi SJ, Ehrenhaus M, Solomon R, et al. The effect of hinge position on corneal sensation and dry eye after LASIK. Ophthalmology. 2003;110(5):1023-9.

3. Rodriguez AE, Rodriguez-Prats JL, Hamdi IM, Galal A, Awadalla M, Alio JL. Comparison of goblet cell density after femtosecond laser and mechanical microkeratome in LASIK. Invest Ophthalmol Vis Sci. 2007;48(6):2570-5.

4. Chao C, Golebiowski B, Stapleton F. The role of corneal innervation in LASIK-induced neuropathic dry eye. The ocular surface. 2014;12(1):32-45.

5. Bower KS, Sia RK, Ryan DS, Mines MJ, Dartt DA. Chronic dry eye in photorefractive keratectomy and laser in situ keratomileusis: manifestations, incidence, and predictive factors. J Cataract Refract Surg. 2015:41(12):2624-34.

6. De Paiva CS, Chen Z, Koch DD, Hamill MB, Manuel FK, Hassan SS, et al. The incidence and risk factors for developing dry eye after myopic LASIK. Am J Ophthalmol. 2006;141(3):438-45.

7. Shoja MR, Besharati MR. Dry eye after LASIK for myopia: incidence and risk factors. Eur J Ophthalmol. 2007;17(1):1-6.

8. Pflugfelder SC. Tear dysfunction and the cornea: LXVIII Edward Jackson memorial lecture. Am J Ophthalmol. 2011;152(6):900-9.

9. Wilson SE, Mohan RR, Hong JW, Lee JS, Choi R, Mohan RR. The wound healing response after laser in situ keratomileusis and photorefractive keratectomy: elusive control of biological variability and effect on custom laser vision correction. Arch Ophthalmol. 2001;119(6):889-96.

10. Wilson SE, He YG, Weng J, Li Q, McDowall AW, Vital M, et al. Epithelial injury induces keratocyte apoptosis: hypothesized role for the 
interleukin-1 system in the modulation of corneal tissue organization and wound healing. Exp Eye Res. 1996;62(4):325-38.

11. Wilson SE, Mohan RR, Mohan RR, Ambrosio R Jr, Hong J, Lee J. The corneal wound healing response: cytokine-mediated interaction of the epithelium, stroma, and inflammatory cells. Progress in retinal and eye research. 2001;20(5):625-37.

12. Gao S, Li S, Liu L, Wang Y, Ding H, Li L, et al. Early changes in ocular surface and tear inflammatory mediators after small-incision lenticule extraction and femtosecond laser-assisted laser in situ keratomileusis. PLOS ONE. 2014;9(9):e107370.

13. Leonardi A, Tavolato M, Curnow SJ, Fregona IA, Violato D, Alió JL. Cytokine and chemokine levels in tears and in corneal fibroblast cultures before and after excimer laser treatment. J Cataract Refract Surg. 2009;35(2):240-7.

14. D'Souza S, Petznick A, Tong L, Hall RC, Rosman M, Chan C, et al. Comparative analysis of two femtosecond LASIK platforms using iTRAQ quantitative proteomics. Invest Ophthalmol Vis Sci. 2014;55(6):3396-402.

15. González-Pérez J, Villa-Collar C, González-Méijome JM, Porta NG, Parafita MÁ. Long-term changes in corneal structure and tear inflammatory mediators after orthokeratology and LASIK. Invest Ophthalmol Vis Sci. 2012;53(9):5301-11.

16. Chao C, Golebiowski B, Zhao X, Chen S, Zhou S, Stapleton F. Long-term effects of LASIK on corneal innervation and tear neuropeptides and the associations with dry eye. J Refract Surg. 2016;32(8):518-24.

17. Nättinen J, Jylhä A, Aapola U, Enríquez-de-Salamanca A, Pinto-Fraga J, López-Miguel A, et al. Topical fluorometholone treatment and desiccating stress change inflammatory protein expression in tears. Ocular Surface. 2018;16(1):84-92.

18. Nättinen J, Aapola U, Jylhä A, Vaajanen A, Uusitalo H. Comparison of capillary and schirmer strip tear fluid sampling methods using SWATH-ms proteomics approach. Trans Vis Sci Tech. 2020;9(3):16.

19. R Core Team. R: A language and environment for statistical computing. Vienna: R Foundation for Statistical Computing; 2020.

20. Szklarczyk D, Gable AL, Lyon D, Junge A, Wyder S, Huerta-Cepas J, et al. STRING v11: protein-protein association networks with increased coverage, supporting functional discovery in genome-wide experimental datasets. Nucleic Acids Res. 2019;47(D1):D607-13.

21. Klepeis VE, Cornell-Bell A, Trinkaus-Randall V. Growth factors but not gap junctions play a role in injury-induced Ca2+ waves in epithelial cells. J Cell Sci. 2001;114(23):4185-95.

22. Crosson CE, Klyce SD, Beuerman RW. Epithelial wound closure in the rabbit cornea. A biphasic process. Invest Ophthalmol Vis Sci. 1986;27(4):464-73.

23. Helena MC, Baerveldt F, Kim WJ, Wilson SE. Keratocyte apoptosis after corneal surgery. Invest Ophthalmol Vis Sci. 1998;39(2):276-83.

24. Kuwabara T, Perkins DG, Cogan DG. Sliding of the epithelium in experimental corneal wounds. Invest Ophthalmol Vis Sci. 1976;15(1):4-14.

25. O'Brien TP, Li Q, Ashraf MF, Matteson DM, Stark WJ, Chan CC. Inflammatory response in the early stages of wound healing after excimer laser keratectomy. Arch Ophthalmol. 1998;116(11):1470-4.

26. Moshirfar M, Welling JD, Feiz V, Holz H, Clinch TE. Infectious and noninfectious keratitis after laser in situ keratomileusis: occurrence, management, and visual outcomes. J Cataract Refract Surg. 2007:33(3):474-83.

27. Levy J, Lapid-Gortzak R, Klemperer I, LifshitzT. Herpes simplex virus keratitis after laser in situ keratomileusis. J Refract Surg. 2005;21(4):400-2.

28. Vesaluoma M, Pérez-Santonja J, Petroll WM, Linna T, Alió J, Tervo T. Corneal stromal changes induced by myopic LASIK. Invest Ophthalmol Vis Sci. 2000;41(2):369-76.

29. Lee BH, MCLaren JW, Erie JC, Hodge DO, Bourne WM. Reinnervation in the cornea after LASIK. Invest Ophthalmol Vis Sci. 2002;43(12):3660-4.

30. Kato T, Nakayasu K, Hosoda Y, Watanabe Y, Kanai A. Corneal wound healing following laser in situ keratomileusis (LASIK): a histopathological study in rabbits. Br J Ophthalmol. 1999;83(11):1302.
31. Linna TU, Pérez-Santonja JJ, Tervo KM, Sakla HF, Tervo TM. Recovery of corneal nerve morphology following laser in situ keratomileusis. Exp Eye Res. 1998;66(6):755-63.

32. Soria J, Durán JA, Etxebarria J, Merayo J, González N, Reigada R, et al. Tear proteome and protein network analyses reveal a novel pentamarker panel for tear film characterization in dry eye and meibomian gland dysfunction. J Proteomics. 2013;78:94-112.

33. Li B, Sheng M, Li J, Yan G, Lin A, Li M, et al. Tear proteomic analysis of Sjögren syndrome patients with dry eye syndrome by two-dimensional-nano-liquid chromatography coupled with tandem mass spectrometry. Sci Rep. 2014;4:5772.

34. Perumal N, Funke S, Pfeiffer N, Grus FH. Proteomics analysis of human tears from aqueous-deficient and evaporative dry eye patients. Sci Rep. 2016;6(1):1-12.

35. Grus FH, Podust VN, Bruns K, Lackner K, Fu S, Dalmasso EA, et al. SELDITOF-MS ProteinChip array profiling of tears from patients with dry eye. Invest Ophthalmol Vis Sci. 2005;46(3):863-76.

36. Versura P, Bavelloni A, Grillini M, Fresina M, Campos EC. Diagnostic performance of a tear protein panel in early dry eye. Mol Vis. 2013;19:1247.

37. Sen DK, Sarin GS. Immunoglobulin concentrations in human tears in ocular diseases. Br J Ophthalmol. 1979;63(5):297-300.

38. Maurya RP, Bhushan P, Singh VP, Singh MK, Kumar P, Bhatia RP, Singh U. Immunoglobulin concentration in tears of contact lens wearers. Journal of ophthalmic \& vision research. 2014;9(3):320.

39. Blalock TD, Spurr-Michaud SJ, Tisdale AS, Gipson IK. Release of membrane-associated mucins from ocular surface epithelia. Invest Ophthalmol Vis Sci. 2008:49(5):1864-71.

40. Sullivan DA, Sullivan BD, Evans JE, Schirra F, Yamagami H, Liu M, et al. Androgen deficiency, meibomian gland dysfunction, and evaporative dry eye. Ann N Y Acad Sci. 2002;966(1):211-22.

41. Versura P, Nanni P, Bavelloni A, Blalock WL, Piazzi M, Roda A, et al. Tear proteomics in evaporative dry eye disease. Eye. 2010;24(8):1396-402.

42. Maimouni S, Issa N, Cheng S, Ouaari C, Cheema A, Kumar D, et al. Tumor suppressor RARRES1-A novel regulator of fatty acid metabolism in epithelial cells. PLoS ONE. 2018;13(12):e0208756.

43. Lindén D, Lindberg K, Oscarsson J, Claesson C, Asp L, Li L, et al. Influence of peroxisome proliferator-activated receptor a agonists on the intracellular turnover and secretion of apolipoprotein (Apo) B-100 and ApoB-48. J Biol Chem. 2002;277(25):23044-53.

44. Arima T, Uchiyama M, Nakano Y, Nagasaka S, Kang D, Shimizu A, et al. Peroxisome proliferator-activated receptor alpha agonist suppresses neovascularization by reducing both vascular endothelial growth factor and angiopoietin-2 in corneal alkali burn. Sci Rep. 2017;7(1):1-11.

45. Gu Y, Li X, He T, Jiang Z, Hao P, Tang X. The antifibrosis effects of peroxisome proliferator-activated receptor $\Delta$ on rat corneal wound healing after excimer laser keratectomy. PPAR Res. 2014. https://doi. org/10.1155/2014/464935.

46. Nakamura Y, Nakamura T, Tarui T, Inoue J, Kinoshita S. Functional role of PPARS in corneal epithelial wound healing. Am J Pathol. 2012;180(2):583-98.

47. Wang Z, Zhang H, Cheng Q. PDIA4: The basic characteristics, functions and its potential connection with cancer. Biomed Pharmacother. 2020:122:109688

48. Jylhä A, Nättinen J, Aapola U, Mikhailova A, Nykter M, Zhou L, et al. Comparison of ITRAQ and SWATH in a clinical study with multiple time points. Clin Proteomics. 2018;15(1):24.

\section{Publisher's Note}

Springer Nature remains neutral with regard to jurisdictional claims in published maps and institutional affiliations. 